\title{
HACIA UNA RESIGNIFICACIÓN TEÓRICA CRITICA DE LA PRACTICA EDUCATIVA
}

\author{
Carlos E. MASSÉ NARVÁEZ; Rocío JUÁREZ GONZÁLEZ \\ UAEMéx (México) \\ edymaster.last@gmail.com,rociojg30@hotmail.com
}

\section{TOWARDS A REDEFINITION THEORETICAL CRITIQUE OF EDUCATIONAL PRACTICE}

Resumen: El texto se apoya en las corrientes pedagógicas críticas que hacen énfasis en la necesidad de resignificar a la práctica educativa, visión que pasa por la redifinición de la educación misma, para distinguirla de la corriente oficial generalmente funcionalista, que coadyuve a la participación en la educación y al concepto mismo de ciudadanía implícito en dichas corriente. Problematización que hacemos al interior de un concepto propio de democracia.

Abstract: The text is based on critical pedagogical trends emphasize the need to give new meaning to the educational practice, a vision that goes through the redefinition of education itself, to distinguish the official generally functionalist that contributes to participation in education and the very concept of citizenship implied in such current. Problematize we do within our own concept of democracy.

Palabras clave: Resignificación. Educación. Práctica Educativa. Participación Ciudadana y Democracia Resignification. Education. Educational Practice. Citizen Participation and Democracy 


\section{Resignifición de la práctica educativa y educación crítica}

El término resignificar en general se plantea para todo profesional formado en disciplinas teóricas, sobre todo cuando éste es formado en las ciencias sociales y, la educación es un objeto de investigación de muchas de ellas. En este caso concreto, la resignificación emerge de la necesidad de llamar la atención a los docentes para que en su práctica educativa, en su profesión, logren una actualización efectiva, a través de ella (de la resignificación en el nivel teórico y práctico, más allá de los cursos que las instituciones de educación superior les proveen, para hacer su propia práctica, más actualizada y más rica, pero sobre todo más motivante y placentera.

En este último sentido, partimos de que el desarrollo del conocimiento posee una inercia constante y, que la aportación de la producción científica aparecida en artículos y en libros en línea, e impresos es importante. En general la información teórica va en aumento constante $y$, a veces es superada por la producción de conocimiento aparecida posteriormente e incluso la que aparece concomitantemente. De ahí que la resignificación viene a ser indispensable para la actualización tanto de la formación teórica del docente, como de dicha práctica educativa en general y, en el nivel teórico y práctico en particular. No obstante, como veremos, algunas aportaciones de la teoría y de la práctica no pierden actualidad, pero progresan con nuevos conocimientos que se suman a una idea de progreso, no necesariamente en la perspectiva sistémica o funcional al sistema, sino que, desde una postura crítica puede contribuir a la independencia de un razonamiento disyuntor que actúa como discurso hegemónico, el que es propio del discurso funcional, sistémico, para tomar distancia de él.

\section{Freire y "la reinvención"}

En ese sentido, el primer autor en que nos apoyamos es en: Paulo Reglus Neves Freire, quien nació en Recife, capital del Estado brasileño de Pernambuco, una de las partes más pobres de este extenso país latinoamericano. Tras haber realizado estudios jurídicos, desarrolló un "sistema" de enseñanza para todos los niveles de educación.

Los fundamentos de su "sistema" se basan en que el proceso educativo ha de estar centrado en el entorno de los alumnos. Freire supone que Ias, (los) educandos, tienen que entender su propia realidad como parte de su actividad de aprendizaje. No basta con suponer que un estudiante sabe leer la frase: "Eva ha visto un racimo de uvas". El estudiante debe aprender a entender a Eva en su contexto social, descubrir quién ha trabajado para producir el racimo y, quién se ha beneficiado de este trabajo. En su publicación más conocida, $P e$ dagogía del oprimido, que está dedicada a los parias de la tierra y a los que se identifican con los pobres, sufren con, y luchan por ellos. En este tipo de trabajo se utilizaban técnicas como los grupos de estudio, grupos de acción, mesas redondas, debates y tarjetas temáticas.

De esta forma, Freire y sus colaboradores empezaron a hablar de un "sistema" de técnicas de educación, del "sistema Paulo Freire" que podía aplicarse a todos los niveles de la enseñanza formal y no formal (Maciel, 1961, citado en Gerhardt, 1983). Debido a ello, destacó el carácter político de la educación y la necesidad de "reinventarla" (resignificarla), en función de las circunstancias históricas (Freire, 1986, pág. 171, citado en Ibidem).

Considerando Freire que él hombre tiene la capacidad innata de razonar, ya había realizado experimentos sobre las reacciones visuales y auditivas de las personas que aprenden a leer y a escribir. En uno de sus primeros experimentos comentó a su empleada doméstica analfabeta una diapositiva en la que figuraba un muchacho y la palabra portuguesa correspondiente (menino). Tras repetir numerosas veces las diferentes sílabas de la palabra y después la palabra entera, Freire observó que María se daba cuenta de cuándo faltaba alguna sílaba y que de esta forma había aprendido que la palabra se compone de sílabas (Freire, 1970b, pág. 9; citado en Gerhardt). 
Freire afirma que un participante, después de sólo haber seguido un curso de alfabetización durante 21 horas, pudo leer artículos periodísticos sencillos y escribir frases cortas. En especial, las diapositivas despertaron gran interés y contribuyeron a la motivación de los participantes. Después de 30 horas de curso (una hora por día, cinco días a la semana), el experimento se dio por finalizado. Tres participantes habían aprendido a leer y escribir. Podían leer textos cortos y periódicos, y escribir cartas. Dos participantes habían abandonado el curso (Freire, 1963a, pág. 19; Freire, 1974, pág. 58; citados en Ibidem.). Así nació el "método" Freire de alfabetización.

Torres, C.A., al hacer un balance analiza la situación de forma un tanto sibilina: "Con gran frecuencia, la competencia técnica en el contexto de reformas educativas políticamente viables y finalmente realizables se contrapone con los principios éticos derivados de las creencias de justicia social y equidad para todos en el contexto de las democracias políticas y económicas. En ocasiones los proyectos de reforma viables políticamente, basados en una ética de corte democrática, carecen de competencia técnica, lo que hace inevitable el fracaso. Por último, proyectos competentes técnicamente y correctos éticamente, pueden no ser fáciles o viables políticamente y permanecer en el reino de las ilusiones, los sueños o el inconsciente de profesionales, maestros y políticos" (citado en Gerhardt).

El interés educativo de Freire se centra en las posibilidades humanas de creatividad y libertad en medio de estructuras político-económicas y culturales opresivas. Su objetivo es descubrir y aplicar soluciones liberadoras por medio de la interacción y la transformación social, gracias al proceso de "concientización", definido como el proceso en virtud del cual el pueblo alcanza una mayor conciencia, tanto de la realidad sociocultural que configura su vida como de su capacidad de transformar esa realidad. Esto supone la praxis, entendida como la relación dialéctica entre la acción y la reflexión. Freire propone un enfoque de la praxis de la educación en el que la reflexión descansa en la acción y la reflexión crítica se basa en la práctica.

Compartimos la idea de reinventar la educación y, que esta sirva para ampliar los procesos cognitivos y la autoconciencia para la liberación. Liberación en términos de des-sujeción mental, y des-formación técnica (liberación de la "razón técnica", o "razón instrumental") en términos de la Escuela de Frankfurt, retomando además, el viejo lema de la Ilustración: "sapere aude" ("atrévete a pensar por ti mismo"). Para ello insistimos en recomendar adoptar la resignificación como forma de actualización cualitativa en términos de actualización teórica, simplemente para re-aprehender a ver y a pensar el contexto críticamente y desde ahí, recuperar y reinventar también algunos aspectos de nuestra cultura. Distinguiendo entre los elemento sanos, libres, positivos, frente a la ideología del sistema oficial y sus poderes hegemónicos.

\section{Educación para la participación ciudadana y la democracia}

Partimos de que la participación ciudadana en general está acotada por el tipo de régimen político. No se puede hablar de democracia desde el mero juego de partidos políticos porque en la mayoría de los casos estos sistemas no contribuyen a la democracia, como es el caso de México. No obstante, hay que ponerse de acuerdo en el uso de conceptos clave que permitan discernir, si una perspectiva educativa puede aspirar o no, a proporcionar elementos para la convivencia en solidaridad que a su vez aspire a una ciudadanía digna. Martha Jalali Rabbani (2001) subvierte el concepto unilineal de educación donde el que sabe, el maestro, el experto, enseña al que no sabe. En una perspectiva de ética comunicativa el espacio de aprendizaje se entiende como una comunidad de comunicación en donde se parte del reconocimiento de la competencia comunicativa de los participantes. La educación para la paz y la educación para la ciudadanía mundial, desde esta perspectiva --nos dice la autora--, siempre es una educación en la reciprocidad, un ejercicio de libertad y reconoci- 
miento (Jalali, 2001, pág.15). Este planteamiento es muy positivo por la visión dialógica entre iguales que se plantea; sin embargo, en términos de una visión así de la educación, no parece factible de ser adoptada por al menos, el Estado Mexicano actual.

La autora aporta elementos valiosos que se identifican con planteamientos hechos hace mucho por otros pedagogos, pero la novedad radica en que se aspira al convencimiento de que una educación para la ciudadanía mundial es factible. Pero sus planteamientos son difíciles de asumir porque creemos que no están contextualizados dentro del ámbito de "lo político". Veamos un ejemplo de sus planteamientos:

"Hablar de ciudadanía mundial -quiero aquí proponer-, es hablar de la inalienable libertad y diversidad humana. Educar, para la ciudadanía mundial, es la toma de conciencia del valor intrínseco de cada uno, lo que nada o nadie puede reprimir (las cursivas son mías). Las he puesto para subrayar el aspecto idílico del planteamiento. Si bien nadie o casi nadie le discutiría a la autora, que esos son derechos humanos innegables, en la vida real no se garantiza su cumplimiento, pues al menos en Latinoamérica son muy socavados. Este estilo de escritura, es muy propio de algunas escuelas filosóficas, pero para ganar en claridad y con realismo, habría que cambiar la expresión nadie puede, por "nadie debe". Este paréntesis que aquí hago, no intenta desvalorizar la obra, pues posee valiosas reflexiones sobre "el despertar de conciencias": ..."educar para el cuestionamiento, para la incesante búsqueda del por qué, de las razones de ser de nuestra realidad y más específicamente, del conocimiento formal" (Jalili; 2001; pág. 17).

La autora apunta a propugnar por un proceso de enseñanza y aprendizaje, conducido por dar y demandar razones, es decir, por el diálogo. Asegura que un proceso así es liberador y más justo, un proceso dialógico, a la inversa de la transmisión monológica del conocimiento, en el cual el llamado "conocimiento objetivo", se pretende irrefutable, pero afirmar que el conocimiento es una verdad objetiva es dejar fuera a las comunidades científicas, las comunidades de reconocimiento donde se hace una investigación (Apel, 1985). En otras palabras, la verdad es siempre intersubjetiva, colectiva y nunca objetiva, individual, como lo presupone una educación fonológica. Apel subraya la necesidad de una comunidad de reconocimiento. Habermas en una concepción similar la concebirá como comunidades de consenso. La educación dialógica se basa en la petición de razones, para compararlas con nuestras propias razones y motivaciones, e inevitablemente transformando esa realidad mientras recreamos sus razones de ser (Jalali, 2001, pág.115).

En la misma perspectiva pero señalando otros aspectos nocivos de la educación "monológica", aunque no la llame así, Querido (2004) señala, que la escuela se ha conformado históricamente en el lugar privilegiado de la abstracción y enseñanza enciclopedista y un "aprendizaje" basado únicamente en la memoria a corto plazo, por lo que propugna por una escuela vinculada muy de cerca a la comunidad, pues conlleva a un cambio actitudinal "incentivando desde los primeros años de escolaridad, actitudes tales como la participación, la solidaridad, el compromiso, la no - violencia y la toma de decisiones, desde el fortalecimiento de la identidad local (ciudad, barrio. Esto implica educar a futuros ciudadanos comprometidos con su contexto, productores, transformadores de su medio: único camino para la formación de una ciudadanía responsable y participativa (pág. 54). (Las cursivas son nuestras).

Decimos que este planteamiento es similar al anterior porque ambos aspiran a una educación para la ciudadanía participativa. El primer planteamiento (de Jalili, 2001), da elementos teóricos para una educación para "la ciudadanía mundial" (en una perspectiva de paz para el desarrollo en términos de intersubjetividad y respeto a la diversidad). En el se- 
gundo (de Querido), se plantea una educación que forma para una democracia participativa. Es decir va más allá que la primera y está fuertemente influenciada por la perspectiva de Freire, pero actualizada y relanzada hacia una práctica ciudadana, se trata al decir de Andrenacci (2004), de "acercar al gobierno a los barrios y empoderar al ciudadano".

Volviendo con Querido, una educación para la democracia participativa debe emprender el siguiente giro pedagógico: Debe pensar en una escuela que trabaje con el contexto, con un currículum que parta de la situación de los alumnos. Debe trabajar con el contexto, para romper con la abstracción, siempre legitimadora de la desigualdad. Tratando de resignificar el rol de la institución escolar rescatando su aspecto transformador y para eso es necesario pensar en una escuela abierta a la comunidad, a la participación de los alumnos y de los padres, al trabajo pedagógico comprometido y situacional.

Estos planteamientos, a diferencia de los de Jalili están basados en la gran experiencia de Porto Alegre, Brasil, pero que por los años de esta tradición, hecho raíces en Argentina, en la que el gran logro ha sido esta fuerte vinculación escuela - contexto; pedagogía situacional. Esto tiene tras de sí un gran impulso basado en la práctica, un gran número de asociaciones civiles, generó a su vez un gran número de proyectos, entre ellos, solo por citar un ejemplo, el de Aprendiendo a Participar, en el que los sujetos de la educación: pedagogos, alumnos y padres de familia propugnaron por un "presupuesto participativo para la educación". Así se articulaba a éste con el proyecto de "aprendiendo a participar":

"Empezamos de a poco a comprender-dice Querido (2004)-, que si bien es de cabal importancia que se abran espacios de participación (llámense Presupuesto Participativo, Centros de Estudiantes, Parlamentos Juveniles...) es esencial acompañar estos espacios con la implementación de propuestas pedagógicas que eduquen, nada más y nada menos, que en el ejercicio de la transformación social. "Aprendiendo a participar" surge con este espíritu, como una manera de profundizar el trabajo del presupuesto participativo ejercitando la toma de decisiones desde los primeros años de la formación escolar. Un proyecto que intenta compartir con los alumnos dos ideas fundamentales a la hora de cambiar la mirada acerca de lo social: 1) La realidad es susceptible de ser transformada, 2) La participación propositiva es la vía para esa transformación" (pág. 54).

Como se ve, una resignificación de lo local a través de una propuesta pedagógica participativa está pensando en una recuperación de la identidad comunitaria. Es decir, una propuesta pedagógica que fomente una relación con el contexto de tono conflictivo, entendiendo el valor pedagógico y social del conflicto, como motor de reestructuraciones cognitivas y políticas respectivamente, con miras a resignificar lo local.

Finalmente nos dice Querido: "Si pretendemos una democracia participativa, estamos obligados a orientar nuestros esfuerzos hacia la construcción de una Pedagogía de la Participación como instrumento para valorar las acciones colectivas y solidarias y el conocimiento del contexto sociocultural. Al abrir las puertas de la escuela se está abriendo también el camino del aprendizaje significativo, de la educación en valores y por lo tanto de una nueva cultura ciudadana (Querido: 2004).

Es importante decir, que estas experiencias de participación ciudadana no son una propuesta teórica como la de Jalili, es el resultado de una necesaria apertura social hacia la auto-organización, en lo que se ha dado en llamar en Latinoamérica "la emergencia de la sociedad civil", lo que en otras altitudes se conoce como el empoderamiento del "tercer sector". Apertura provocada desde la propia ciudadanía para auto-defenderse de los excesos de los regímenes militares, o los del Estado Nacional en decadencia, también conocido como estado neoliberal. En donde el Estado ha perdido alto porcentaje de soberanía frente al gran capital transnacional y nacional impactando en el alza de la pauperización de la sociedad. 
Creemos que también es importante decir, que aún con las similitudes existentes entre nuestros países, no habría que creer que estas interesantes propuestas pueden ser implementadas cuales modelos a copiar, sino que su posible implementación, dependería del nivel de la práctica sociopolítica de la ciudadanía. La cual para el caso de México es sumamente baja (si tomamos en cuenta la corrupción del sistema electoral y nuestra síntesis diagnóstica de la educación en general: el sistema educativo mexicano-- que de por si no resiste el análisis crítico de sus contenidos y objetivos, una educación bancaria (como la llamara Freire). La enseñanza se ha caracterizado desde hace mucho tiempo por ser predominantemente verbalista y tradicional, propiciando una actitud receptiva y pasiva del alumno, convirtiéndolo en un ser estático, apático, carente de creatividad e iniciativa, depositario de una visión unilineal del mundo.. Un gran número de ejemplos de los frutos de esa participación con base en dicha perspectiva pedagógica, pueden verse a lo largo del texto colectivo de (Romero: 2004).

Por todo lo anterior, asumimos la necesidad de preguntarnos ¿Cómo creemos que debe ser la educación (la práctica educativa) en el umbral de la postmodernidad? En uno de sus múltiples trabajos de síntesis, Postmodern Social Theory George Ritzer (1997), afirma que «hay muchas formas de caracterizar la diferencia entre los mundos moderno y postmoderno, pero, como ejemplo, una de las mejores es la diferencia en puntos de vista sobre si es posible encontrar soluciones racionales (...) a los problemas de la sociedad». En otras palabras, la época postmoderna, la postmodernidad, desespera de la razón, la sociedad pierde la fe en la razón. ¿Qué rasgos caracterizan la cultura postmoderna (la cultura de un mundo, recordemos, que ha dejado de fiarse de la razón)? A juicio de Ritzer (1997: 8):

"El desafío a los límites modernos. En otras palabras, crítica del sistema de categorías que ordenaba la sociedad moderna. Se rechazan definiciones, barreras entre disciplinas (académicas y no académicas), se pone en tela de juicio la diferencia entre realidad y ficción. No es simplemente una crítica al vocabulario moderno y a una forma de ordenar el mundo".

En nuestra opinión, si el lema Sapere aude (atrévete a pensar por ti mismo) fue el grito de guerra de la clase que se encaramaba en el poder, creando al estado burgués, ¿Qué nueva frase generarían las nuevas generaciones desencantadas del mundo, como ariete para hacer otra gran transformación? Pregunta que sólo dejamos planteada como reflexión obligada a las nuevas generaciones

Consideramos con respecto a nuestra práctica educativa que, como docentes optamos por una práctica educativa reflexiva con base en la resignificación, de nuestra formación teórica y práctica en nuestra propia práctica educativa, pero es necesario advertir que, dicha resignificación se basa en una práctica educativa y reflexiva sustentada en la investigación acción, la que esbozamos a continuación con base en la obra de Pedroza (2011).

\section{Breve resumen de una propuesta para la autorreflexión en la práctica educativa}

He dejado este último espacio para presentarles una síntesis muy apretada de lo que, la práctica educativa debe hacer para continuar con el objetivo de la emancipación del ser humano. En tal sentido, a continuación presentamos algunos elementos sobre la práctica educativa reflexiva, sustentada en la investigación-acción.

El estudio de la práctica educativa se propone desde el abordaje metodológico de la investigación-acción. Porque en esta relación se construyen la significación y la resignificación de la acción docente en sus distintos roles dentro de la institución educativa. La práctica educativa supone un movimiento circular entre teoría y práctica: no se concibe la práctica sin sustento teórico ni teoría sin referencia a la práctica. Aunque no siempre 
se reconoce la teoría que está implícita en la práctica, el reconocimiento depende del tipo de movimiento circular que puede ser vicioso o virtuoso. El movimiento circular vicioso carece de resignificación porque en la práctica educativa prevalecen rasgos asociados con la improvisación, la tradición, el amateurísmo, la reproducción y el tecnicismo. El movimiento circular virtuoso posee resignificación porque en la práctica educativa está presente un continuo de autorreflexión.

En ambos círculos se presentan saltos cualitativos entre práctica y teoría; sin embargo, no son construidos de la misma forma: en el círculo vicioso la construcción de los saltos cualitativos es espontánea o por tanteo, la necesidad mediata obliga a buscar soluciones a los imprevistos que se presentan sin pretender la emancipación de cánones tradicionales; y en el círculo virtuoso la construcción de los saltos cualitativos es planeada, organizada y, se construye a través de metodologías cualitativas, la finalidad es la emancipación de la conciencia reflexiva.

En este punto el autor se ocupa de los círculos virtuosos. Identifico en este tipo de círculo -nos dice Pedroza (2011) la existencia de un triple proceso entre la relación práctica (P) y teoría $(\mathrm{T})$. El triple proceso que propongo --continúa diciendo el autor--, conduce a la reformulación articulada de PT (práctica-teoría) a través de un conjunto de operaciones lógicas y de consideraciones conceptuales, la triada se desarrolla de la manera siguiente: el primer proceso de aprehensión y construcción de significaciones Ps-(Ts+Tc)- $\operatorname{Tr}$ (conocer); en donde "Tc" es la Teoría conocida, el segundo proceso de desarrollo y evaluación de significaciones y resignificaciones $\mathrm{Tr}$ - (Pr-Pvr)-Pvr $>$ (valorar); y tercer proceso de revaloración, síntesis y aporte Pta (emancipar), Gráficamente se representa así:

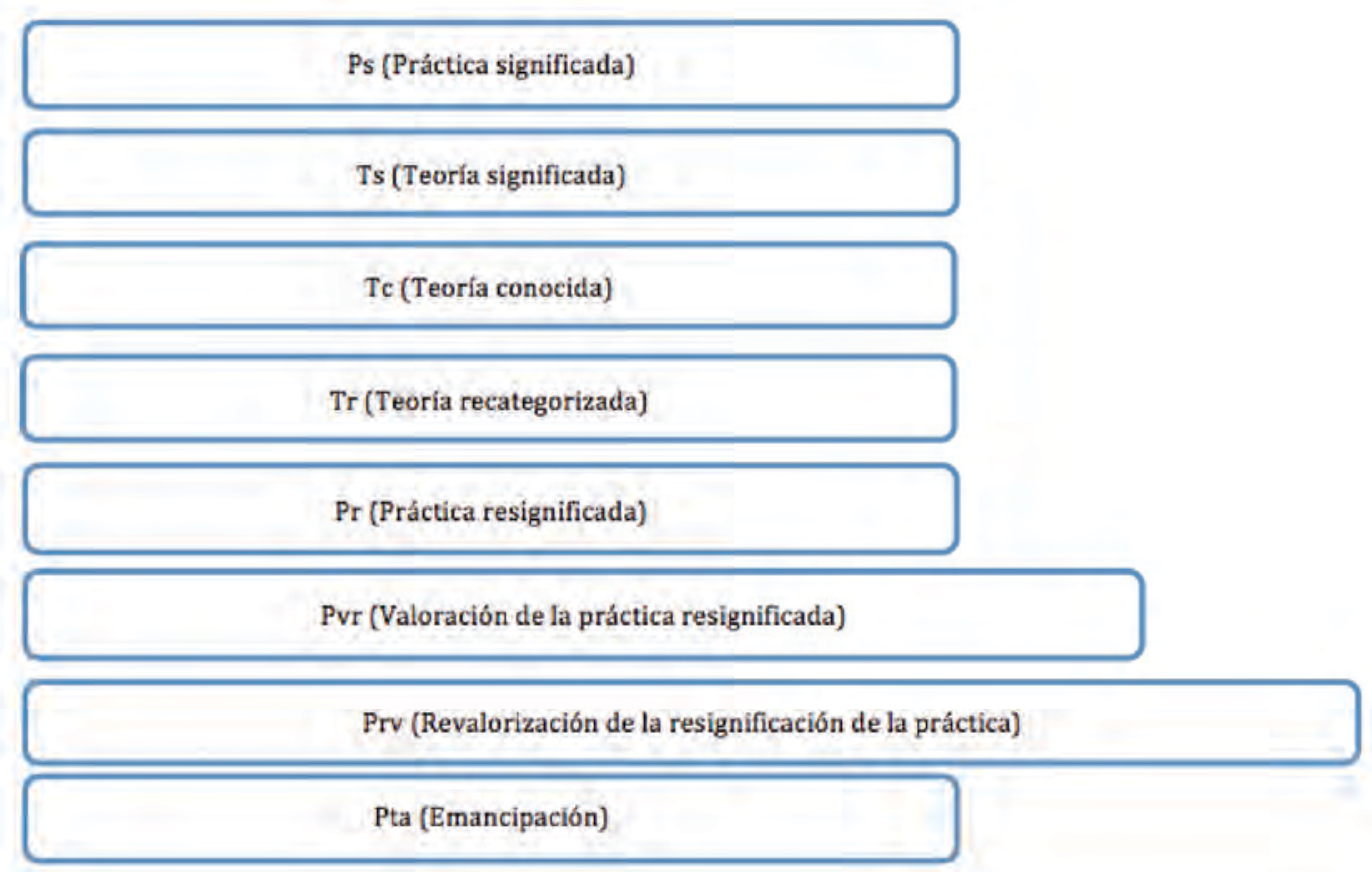

Esquema I

Desenvolvimiento de las figuras práctica-teoría

Fuente: Elaboración personal del autor 
En la ilustración se plasman los tres procesos, en cada uno de ellos se observan distintos momentos que están separados con guiones y representados con letras. En cada proceso están siempre presentes la práctica y la teoría, siendo cualitativamente diferentes, su cualidad se nota con el adjetivo que sumen tanto la teoría como la práctica. Los adjetivos que reciben las figuras de la relación entre práctica y teoría son los siguientes:

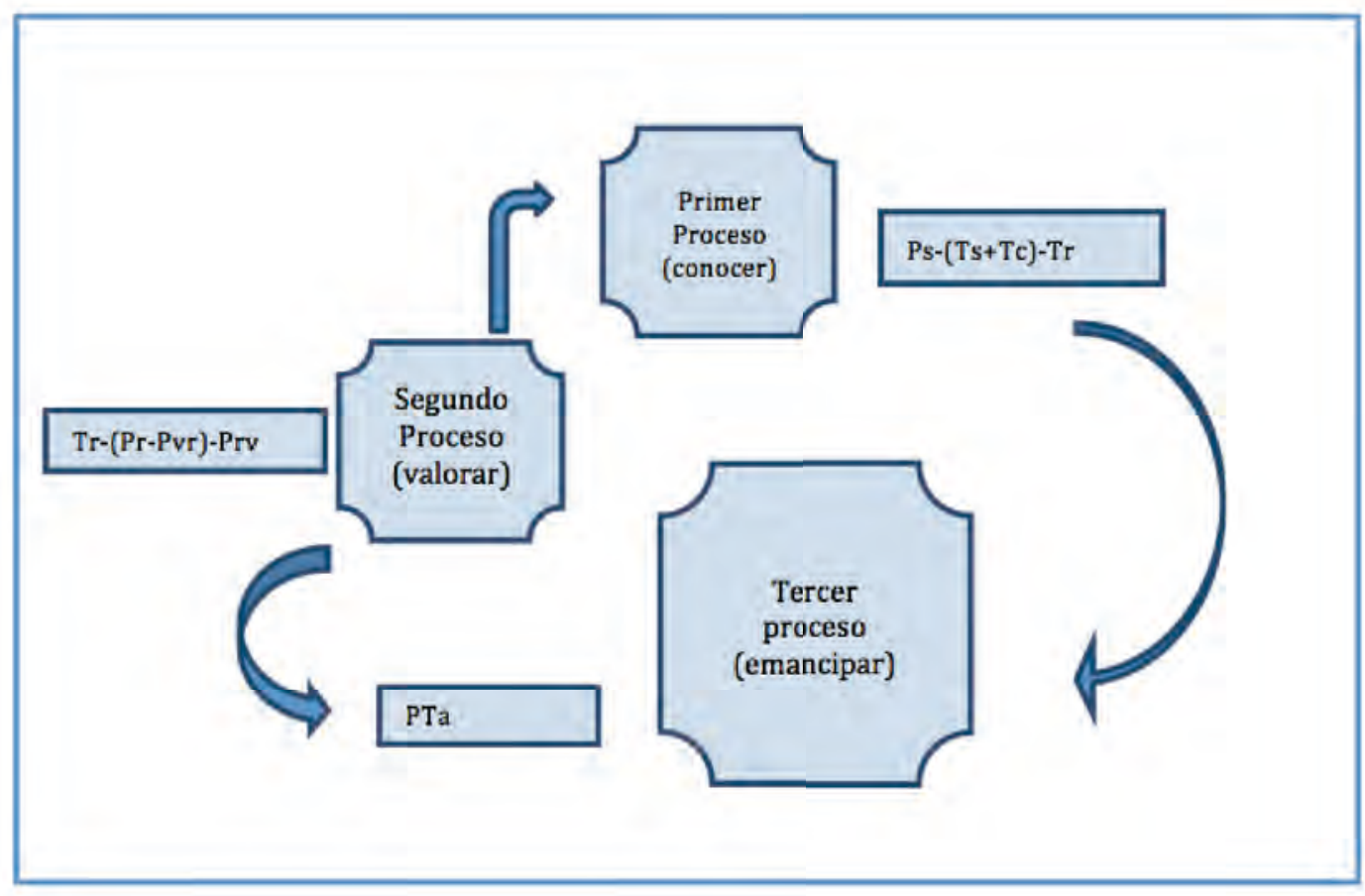

Esquema 2

Proceso de la relación práctica teoría

Fuente: Elaboración personal del autor

El primer proceso lo conforman tres momentos. El primer momento es Ps, se define como la práctica significada. Consiste en aprehender el significado de la propia práctica educativa en el contexto (cotidianeidad), en el texto (la acción docente) y en el pretexto (subjetividad e intencionalidad), Considerando que la dinámica entre contexto, texto y pretexto se da a partir de la existencia de objetos, sujetos e interrelaciones que permean al docente.

El significar la práctica educativa es un proceso de semiosis porque se trata de cualidades, existencia y de pensamiento. Este planteamiento lo sustenta el autor en la teoría de Peirce, en particular con el argumento de que realidad y conocer se encuentran en un mismo universo.

Sustentamos que la práctica docente sólo se puede conocer sí se aprehenden sus significados que la constituyen en la realidad misma en la que se observa y registra. El docente es observador de sí mismo, conoce los significados de sus acciones al indagar sobre sus intencionalidades a través de posicionarse en el terreno de la subjetividad.

El segundo momento del proceso inicial es Ts, se define como la teoría significada. Consiste en categorizar la experiencia, en trabajar a través de los registros de la observación -y con otras fuentes de conocimiento de la propia práctica educativa- en la identificación, reflexión y comprensión de los aspectos conceptuales derivados de la experiencia. Esto permite conocer la problemática o problemáticas de la propia práctica educativa, de velar las intencionalidades y conocer los elementos constituyentes de la práctica. 
Se capta a la experiencia en su complejidad, en las interacciones de la subjetividad del propio docente en su relación con los otros (intersubjetividad) y con relación a sí mismo (intrasubjetividad). Se trata de observar a la práctica educativa tal y como es, para después singularizar al docente en su práctica como que si es uno mismo y, después media la interpretación, el conocimiento como comprensión de los significados de la práctica docente.

En el segundo momento del proceso inicial se ubica también Tc, es la teoría existente en torno a las categorías construidas de la propia práctica docente, es el insumo del diálogo teórico, es indagar que tanto se conoce o se desconoce conceptualmente de la propia singularidad.

En el tercer momento del proceso inicial se ubica Tr, es la teoría recategorizada o resignificada. La teoría se concibe como un proceso no como algo acabado, que va de la acción a la conceptualización y viceversa. Es un continuo comparativo entre Ts y Tc, con la finalidad de resignificar el conocimiento dentro de la realidad misma. Este planteamiento retoma aspectos del aporte de Anselm Strauss y Glaser, quienes generaron la Grounded Theory. Patricia Gaytan (2008), ubica los presupuestos de estos autores en los siguientes términos:

"Para la rama del interaccionismo en la que se sitúa Strauss, los sujetos son portadores y constructores de lo social, de tal forma que es posible conocer la realidad social a partir de las personas que la conforman. De tal manera que sus acciones son explicables mediante la comprensión del sentido, de los significados asociados a ellas y que son compartidos por un grupo. Esos significados pueden ser explícitos y comprensibles para los actores sociales, o pueden ser implícitos y asequibles al investigador por medio del análisis de los discursos y de los hechos. De modo que es posible objetivar lo subjetivo. Es decir desentrañar los significados de la prácticas, las creencias y las valoraciones a partir del entorno cultural en el que se gestan las situaciones que constituyen el objeto de estudio del sociólogo" (p. 6)

Pedroza resalta el proceso de objetivar lo subjetivo, que señala Gaytan, porque ahí reside la construcción categorial de los significados, media el trabajo conceptual entre los momentos que identifico como Ts y Tc, que Strauss y Glaser, denominan teoría sustantiva y teoría formal, la primera asociada a lo empírico y la segunda a lo desarrollado en un campo del conocimiento.

En la argumentación de nuestro autor, la teorización de la práctica educativa supone la parte sustantiva que ubica en la intuición y la experiencia y la parte formal, la coloca en la reflexión conceptual de campos de conocimiento específicos. Por esta razón, la teoría significada, es la incorporación de significados, a los significados antes aprehendidos.

En el segundo proceso, conformado por tres momentos, parte del resultado del primer proceso, de $\operatorname{Tr}$ (primer momento). La teoría significada se objetiva en el diseño de un modelo de intervención de la práctica educativa. Consiste en construir la solución del problema; en caso de que se asuma una concepción técnica de la intervención de la práctica educativa o, en trabajar sobre lo inconsciente de la propia práctica educativa. En el caso de que se asuma una concepción clínica de la docencia, ello significa concientizar la acción docente. En ambos casos se trata de construir el método que permitirá profesionalizar el trabajo docente con miras a mejorar, cambiar, transformar y/o innovar.

En el segundo momento del segundo proceso Pr, significa práctica resignificada. Es la práctica que ha sido intervenida con nuevos significados. Representa el momento de revitalización puntual de la práctica educativa. Se realiza el seguimiento técnico y/o de la subjetividad de cada una de las acciones, con el propósito de superar la problemática y de conocer nuevos significados. 
El tercer momento del segundo proceso es Pvr, se define como la valoración de la resignificación de la práctica. Como se trata de un proceso intencional consiente se evalúa continuamente, con la finalidad de ir ajustando los significados.

El segundo proceso concluye con Prv, se define como la revalorización de la resignificación de la práctica.

El tercer proceso es PTa, se define como la síntesis de la relación entre teoría y práctica a lo largo de los dos procesos descritos. Representa el momento de aporte, la contribución al Estado del Conocimiento a partir de la singularidad estudiada, como práctica educativa intencional y objetiva.

Y una vez expuesto el contenido de la relación teoría-práctica y de ubicar los diferentes momentos que atraviesa el sujeto (docente) de la significación y la resignificación surge una pregunta: ¿cómo plantear el trabajo de la investigación-acción a partir de la forma en que se desdobla la práctica y la teoría y sus vínculos que desarrollan? La respuesta se encuentra, expuesta en la obra del autor en cuestión, aquí, por último sólo exponemos la brevísima parte que a ella dedica en sus "Reflexiones finales".

Pedroza plantea que en la conexión entre los procesos de la relación práctica-teoría que acabamos de ver y, las etapas de la investigación-acción, se conoce la significación y se construye la resignificación de las acciones de la práctica educativa. Existe una comunicación entre acción e indagación que puede ser leída desde los aportes de Peirce. Coincidimos con Elizondo (2006: 41), citado por Pedroza 2011), al señalar que en la obra de Peirce podemos leer una teoría de la significación que puede asumirse como una teoría del conocimiento, como lógica de indagación de conceptos y símbolos. En este sentido -señala Elizondo (2006:106)-- el pragmatismo de Peirce, se puede concebir como un método para indagar los significados. Esto se puede leer directamente en Peirce:

"Entiendo --dice Peirce-- el pragmatismo como un método para indagar los significados, no de todas las ideas, sino únicamente de aquellas que llamo "intelectual concepts". (...) Estos conceptos intelectuales que son los únicos signos - con carga (sign-burdens), que son denominados adecuadamente como 'conceptos', esencialmente llevan consigo alguna implicación concerniente al comportamiento general de, ya sea, un ser consciente, o de un objeto inanimado. Y refiere a la posibilidad de que tal conducta se lleve a cabo bajo ciertas circunstancias. Pero el pragmatismo sostiene el significado total del predicado de un concepto intelectual, se encuentra contenido en la información que, bajo todas las circunstancias imaginables de algún tipo, el sujeto del predicado se comportará de una manera general" (Elizondo, 2006, p.106, citado por Pedroza, 2011).

Los significados -podemos leer de la cita-(continúa Pedroza) que los conceptos intelectuales tienen implicación en el comportamiento de un ser consciente o de un ser inanimado, lo que implica que la conducta se realice en ciertas circunstancias que puede ser reconocida a través de la indagación de sus significados. En esto encontramos -lo que otros autores ya han señalado- una respuesta antropológica de lo que el hombre es. En Peirce, el hombre es signo; y el signo, se concibe como un mecanismo de comunicación que transmite un significado desde una conducta al indagador. El hombre al definirse como signo podemos conocerlo por la interpretación de los significados de su acción. O como señala Barrena y Nubiola. El hombre es un sujeto histórico con todo lo que esto representa, donde a futuro es un mapa de signos abiertos a su interpretación. Textualmente estos autores expresan de esta forma su planteamiento:

"El sujeto es un conjunto de posibles relaciones que se van actualizando en el tiempo y que requieren para su expresión de un organismo que tiene una existencia temporal. El sujeto-signo es un ser histórico y encarnado, 
culturalmente determinado, embebido de su tiempo y lugar (...) Estas características de la persona humana, su apertura, temporalidad, incompletitud y continuidad hacen que el hombre pueda siempre crecer. El hombre, como signo, está inmerso en un proceso de semiosis universal e infinito por el que ese signo va dando lugar a otros nuevos indefinidamente. Los signos hacia el futuro" (Barrena y Nubiola, 2006, p.4; citados por Pedroza, 2011).

Esta concepción de sujeto que retoma Pedroza (2011) por la posibilidad de poder encontrar sus características en la práctica educativa. El docente es un sujeto que actúa, se manifiesta en conductas, comportamientos y relaciones, que mantiene significados de los que debe dar cuenta. Por esta razón, en esa conexión entre práctica y teoría. Podemos conocer y teorizar esos significados y sus resignificaciones en un proceso abierto hacia el futuro. No se agota la investigación-acción de la práctica educativa con el logro del objetivo de un mejoramiento, cambio o transformación, es un continuo la semiosis universal de las acciones y sus intencionalidades.

El docente en su tarea de indagar sobre sí mismo es un sujeto dinámico en búsqueda constante de sus significaciones que le preceden, esta idea es parecida a la expresada por Ibañez $(1985,269)$ :"Sólo un investigador como sujeto en proceso puede perseguir una verdad que siempre se le escapa”. El docente como investigador de su práctica es un sujeto en construcción. En esta construcción el sujeto se define con relación a su significante y el significante es lo que representa a un sujeto para otro significante; el significante emana al sujeto de su significación (Lacan 2007). Miller (2006; citados por Pedroza 2011) expresa al respecto, que el sujeto es efecto del significante, lo que significa que el significante habla de él antes de que él hable, el sujeto no es nada sin el significante.

El sujeto de la práctica educativa representa en lo "real" un "producto" del significante, que ante sí como otro, representa otro significante. Por esto, no bien termina una categorización de sus significaciones cuando está construyéndose a partir de su significante que puede comprenderse o interpretarse como una resignificación. En esto reside la importancia del estudio de la práctica educativa a través de la investigación-acción: la construcción del sujeto a partir de su significación y de su relación con el otro como otro significante.

La profesionalización de la práctica educativa es un proceso de resignificación cuya finalidad es la emancipación del docente de sus ataduras tradicionales que lo anclan a obstáculos pedagógicos. Profesionalizarse el docente significa asumir una coincidencia inacabada y comprometida con la filosofía educativa, de una teoría crítica de la educación que sustenta la relación práctica-teoría.

Nota aclaratoria de los autores: Sin ninguna duda, esta teoría de Peirce no guarda ninguna semejanza con la noción vulgar de pragmatismo, que connota una burda búsqueda del beneficio así como la conveniencia política. En cambio, el pragmatismo de Peirce es un método de experimentación conceptual, hospitalario para la formación de hipótesis explicativas, y propicio para el uso y la mejora de la verificación. Típico de Peirce es su interés en la formación de hipótesis explicativas como fuera de la alternativa fundacional habitual entre el racionalismo deductivista y el empirismo inductivista, aunque Peirce fue un lógico matemático y un fundador de la estadística.

\section{Conclusiones}

Finalmente, para una educación democrática nos apoyamos en el concepto fuerte de Habermas de democracia como lo plantea en Facticidad y Validez (...) en el que plantea que, un síntoma de existencia de democracia sólo podrá logarse cuando la sociedad realmente representada pueda darse a sí misma las leyes que considera le permitirán vivir en armonía menos constreñida por los dictados del poder . Cabe con él, apuntar un primer sentido de 
educación democrática como aquella que se imparte a todos por igual, y de ahí que se entienda por democratización de la enseñanza, abrir las instituciones educativas a todos los miembros de la sociedad, lo que desde los supuestos antropológicos y políticos enunciados -implicación recíproca de libertad e igualdad parece evidente--. Ya la burguesía revolucionaria en el Siglo XIX, consciente de la contradicción existente entre su consigna de libertad, igualdad y fraternidad -fundamento intocable de toda noción fuerte de democracia- y, la existencia de una propiedad repartida muy desigualmente, establece, la educación como principal factor compensatorio de la desigualdad social, pero ésta no puede quedarse anquilosada en un práctica educativa que no se actualiza y que se somete a los dictados de la visión funcionalista oficial de la educación. Ésta sólo podrá ser útil a los cambios evidentes que las sociedades requieren, con base en una resignificación profunda de su práctica educativa, reflexionando críticamente hacia los elementos con los que fueron formados, a través de resignificar (actualizar) sus conocimientos teórico-educativos y contrastarlos con sus realidades locales, en el contexto de una globalización neoliberal, la que es depredadora en sí misma del pensamiento común de los pueblos, situación que sólo puede ir cambiando, gracias a una educación de calidad, es decir crítica e igual para todos. De una práctica educativa crítica ha de provenir retomar y resignificar el derecho a una enseñanza igual para todos, que elimine o reduzca al máximo el sentido aristocrático de la enseñanza, en el doble sentido de impartirse sólo a unos cuantos, y con unos contenidos que pretendan perpetuar el status privilegiado de unos pocos. La que además ha de estar a cargo del Estado, que conlleve una enseñanza con métodos y contenidos democráticos.

\section{Bibliografía}

Apel, K-O. (1995). Teoría de la verdad y ética del discurso, Paidos, Barcelona,

Cardona, Patricia "La educación básica escolar adolece de un vacío conceptual y de eurocentrismo". En Uno Más Uno, México, 29 de mayo de 1985.

Freire, Paulo. La educación como práctica de la libertad. Ed. Búsqueda, CEDALEC, 1985.

Gerhardt, H. P. (1983). "Paulo Freire", (Pensadores de la Educación), en Revista Perspectivas de la Educación, Vol. 2, UNESCO.

Jalali R. Martha. (2001). La educación para la ciudadanía mundial. Reconociendo la unidad y realizando la diversidad humana, México, ed. UAEM.

Pedroza, F. René (2011). La investigación-acción en la práctica educativa reflexiva, ed. Colegio de Investigadores en ciencias de la educación/ed. Lulu: http://www.lulu.com/shop/renépedroza-flores/la-investigación-acción-en-la-práctica-educativa-reflexiva/paperback/product-14680709.html

Ritz, George (1997). The postmodern social theory, Usa, McGraw-Hill Higher Education.

\section{3}

